\title{
Heike Mundzeck, Journalistin
}

\author{
djb-Frauen machen Karriere - wir stellen sie vor
}

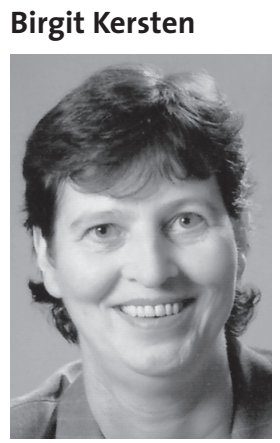

Vorstand Regionalgruppenbeirat; Vorsitzende der Regionalgruppe Oldenburg im djb; Rechtsanwältin und Vereidigte Buchprüferin, Brake
Das Interview führte Birgit Kersten, Vorstand Regionalgruppenbeirat und Vorsitzende der Regionalgruppe Oldenburg, im April 2008.

Sie sind in der Kriegs- und Nachkriegszeit aufgewachsen. Ihre Mutter hat über Jahre die Familie ernährt. Wie fanden Sie das und wie empfanden Sie dann die „Zurück an den Herd“-Politik der 5oer Jahre?

Da ich nichts anderes erlebt habe, empfand ich es als völlig normal, dass meine Mutter berufstätig war und sein musste - mein Vater kam erst 1948 aus der Kriegsgefangenschaft zurück. Ich habe noch zwei jüngere Geschwister und musste schon als Kind einige häusliche Aufgaben übernehmen, d.h. ich bin sehr früh selbständig geworden. Als mein Vater dann beruflich wieder Fuß fasste, hat meine Mutter ihren Beruf aufgegeben. Natürlich finden Kinder, die eine Mutter zuhause haben, das auch ganz schön, aber ich konnte sehen, dass sie selbst darunter litt. Sie wäre lieber weiter berufstätig gewesen. Doch damals, in den 50er Jahren, ging das einfach nicht. Ein Rechtsanwalt, der eine arbeitende Gattin hatte, wurde verdächtigt, seine Familie nicht ausreichend versorgen zu können. Und in diesem Sinne hieß es: „Meine Frau braucht nicht zu arbeiten." So hat sie sich gefügt.

Sie haben 1957 mit dem Jurastudium begonnen. Warum wählten Sie Jura? Wie viele Kommilitoninnen hatten Sie? Wie war Ihr Verhältnis zu Professoren und Studenten?

Ich wollte immer Journalistin werden und hätte selbst am liebsten Germanistik oder Literaturwissenschaften studiert, aber mein Vater sagte: „Studier' mal was Ordentliches. Bei den Juristen gibt es noch nicht so viele Frauen, und damit kannst du immer etwas anfangen." Er hatte sicherlich die Hoffnung - er war ja Rechtsanwalt -, dass ich dann doch genug Freude an der Juristerei finden würde, um später sein Anwaltsbüro zu übernehmen, was ich aber nie im Sinn hatte.

Als ich anfing, Jura zu studieren, gingen auch wir Studenten noch sehr formell miteinander um. Wir haben uns damals z.B. gesiezt. In einem Hörsaal mit 100 Hörern saßen vielleicht drei Frauen. Mit einer habe ich mich dann angefreundet. Es waren aber auch männliche Kommilitonen da, mit denen man sich gut verstand.
Heike Mundzeck, geb. 1938 in Hamburg, studierte von 1957 bis 1963 Rechts- und Staatswissenschaften an den Universitäten in Marburg, Innsbruck, Freiburg und Hamburg mit dem Abschluss 1. Staatsexamen.

Von 1963 bis 1964 absolvierte sie ein Volontariat bei der Tageszeitung DIE WELT in Essen, Berlin und Hamburg.

Von 1964 bis 1970 war sie Redakteurin des Kulturteils der WELT. Seit 1971 arbeitet sie freiberuflich als Journalistin für Tageszeitungen, Zeitschriften, den Rundfunk und als Buchautorin.

Seit 1975 ist sie Filmemacherin (über 100 Fernsehproduktionen für ARD, ZDF, die Bundeszentrale für politische Bildung und andere Bildungs-Institutionen und Verbände).

Von 1994 bis 2003 war sie Vorstandsmitglied der Hamburgischen Anstalt für neue Medien. Seit 1995 ist sie Fernsehproduzentin.

Heike Mundzeck ist Mitglied in verschiedenen beruflichen Verbänden und Jurys.

In den djb ist Heike Mundzeck 1976 eingetreten. Sie war zwei Jahre Mitglied des Bundesvorstandes.

Zu den Professoren gab es eigentlich keinen Kontakt. Die älteren Herren etwa konnten kaum akzeptieren, dass auch Frauen im Hörsaal saßen. Professor Rape zum Beispiel, der damals schon vor der Emeritierung stand, sagte fast regelmäßig, wenn er seine Vorlesung begann: „Guten Tag meine Herren. Die hier anwesenden Damen gehören eigentlich ins Haus und an den Herd. “Dann klopften manche Studenten. Es gab aber auch welche, die schon mal ein leises „Buh“ wagten. So war noch die Einstellung in der damaligen Zeit. Uns junge Mädchen hat das nicht weiter gestört. Wir haben uns gesagt: wir sind jetzt hier und werden Jura studieren.

Gab es Dozentinnen? War an Juraprofessorinnen überhaupt zu denken, also wäre eine wissenschaftliche Karriere eine Option gewesen?

Es gab Dozentinnen, es gab in Hamburg auch eine Professorin, Frau Prof. Dr. Brauneck. Eine wissenschaftliche Karriere war für Frauen schon möglich. Es haben ja auch Frauen aus meinem Studienjahrgang wissenschaftlich Karriere gemacht. Aber für mich kam das gar nicht in Frage. Ich war froh, als ich das Studium hinter mir hatte und nun endlich, endlich Journalistin werden konnte. Als mein Vater 
mich nach dem Examen erwartungsvoll ansah, da habe ich mich zwar noch zum Referendariat angemeldet, aber gleichzeitig nach einen Volontärsplatz bei einer Zeitung gesucht. Mit etlichen Schwierigkeiten habe ich den dann auch bekommen, und zwar schneller als einen Referendarplatz, denn es gab damals lange Wartezeiten. Und nun war klar, dass ich in den Journalismus ging.

\section{Wie wohnten Sie? Machten sich Ihre Eltern Sorgen um Sie?}

Ich habe in vier verschiedenen Städten studiert, in Marburg, Freiburg, Innsbruck und Hamburg. In den auswärtigen Städten hatte ich eine so genannte „Bude“. Die kostete 35 bis 50 Mark: ein möbliertes Zimmer mit einer Wirtin, die immer ganz streng guckte, ob da auch nichts Unrechtes passierte, ob man seine Sachen in Ordnung hielt und ob auch nach 22 Uhr kein Herrenbesuch im Zimmer war, was für uns, die wir ja zumeist noch sehr brav erzogen waren, ohnehin nicht in Frage kam. Also, meine Generation war da noch nicht so locker, das hätten wir damals höchst unmoralisch gefunden.

Sorgen machten sich meine Eltern um mich überhaupt nicht. Sie hatten auch keinen Grund dazu, denn ich war wirklich ihr Erziehungsprodukt in dem Sinne, dass ich wusste, wie ich mich zu verhalten hatte. Ich durfte deswegen auch schon in sehr jungen Jahren, also mit 13, 14, mit einer Freundin mit

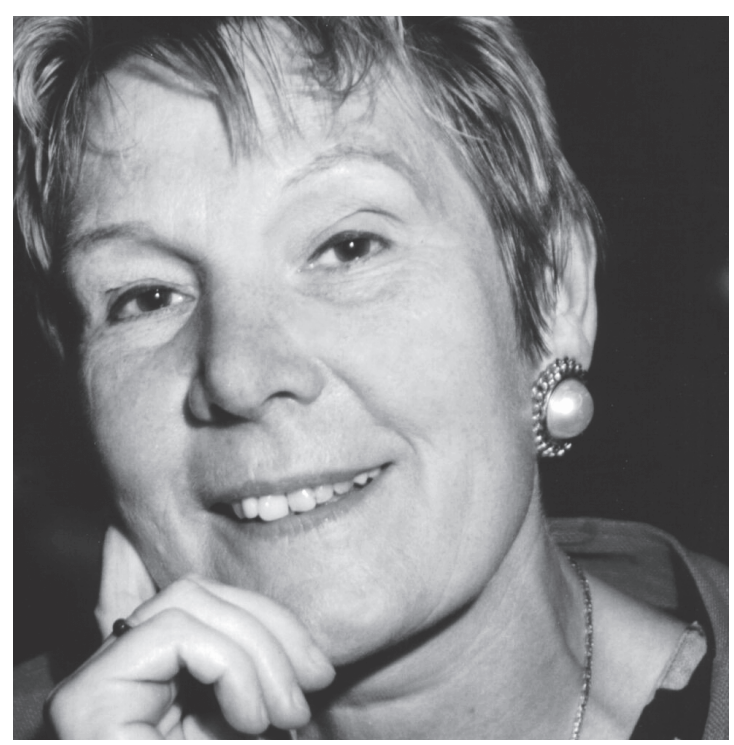

setzt. Dann sollte man wechseln, was heute allerdings nicht mehr so einfach geht wie früher. Wir hatten ja damals eine ziemlich bequeme Lebenssituation. Man konnte sich bei jeder Universität anmelden. Man schrieb sich einfach ein. Es gab keinen Druck, Scheine zu einem bestimmten Zeitpunkt zu machen. Es waren wirklich ganz andere Bedingungen als heute, wo die jungen Leute unter viel größeren Zwängen stehen.

\section{Was halten Sie davon, dass die juristische Ausbildung immer schmalspuriger wird, also die „Volljuristin“/der „Volljurist" verschwinden soll?}

Ich bin ja selbst keine Volljuristin. Selbstverständlich braucht man für bestimmte Arbeitsprozesse unbedingt die Volljuristin oder den Volljuristen. Wer später Richter oder Rechtsanwalt werden will, auch ein Intendant oder Justitiar in einem Sender, sollte Volljuristin oder Volljurist sein. Andererseits gibt es viele Berufe, in denen juristische Kenntnisse sehr hilfreich sind, aber das zweite Staatsexamen nicht unbedingt nötig ist. Ich habe es nicht gebraucht.

Es gab damals ja noch nicht die später wieder abgeschaffte - einstufige Juristenausbildung, bei der man während des Studiums bereits praktische Erfahrungen sammeln konnte. Ich hätte sie sonst sicherlich gewählt. Mir hat die direkte Verbindung von Theorie und Praxis immer mehr gelegen.

dem Fahrrad durch ganz Deutschland fahren und dann später ins Ausland. Ich habe ihnen dieses Vertrauen und diese Großzügigkeit immer hoch angerechnet, sie haben mein Selbstvertrauen sehr gestärkt.

\section{War es üblich, die Universität so oft zu wechseln, wie Sie es getan haben? Halten Sie örtliche Flexibilität und Uni-Wech- sel für notwendig?}

Ich hatte das Glück, in einer mittelständischen Familie aufzuwachsen. Mein Vater fand es richtig, dass ich mehrere Universitäten kennen lernte, er hat selber an verschiedenen Universitäten studiert. Es war also Chance und Luxus zugleich. Ich finde es vorteilhaft, wenn Studenten die Möglichkeit zu einem Auswärts-Studium bekommen, aber ich denke, aus lerntheoretischen Erwägungen ist es nicht unbedingt notwendig, es sei denn, das gewählte Studienfach ist an einer Uni nicht gut be-
Besonders wichtig finde ich jedoch, dass Juristen in ihrem Studium psychologische Kenntnisse erwerben. Sie sollten einiges über menschliche Verhaltensweisen und ihre seelischen Hintergründe wissen. Man sieht das ja an der Entwicklung der Mediation, die ich für sehr vernünftig halte, zum Beispiel bei Scheidungen, bei Nachbarschaftsstreitigkeiten oder im Gesellschaftsrecht. Man kann vieles außergerichtlich regeln. Prozesse dauern oft Jahre und bringen auch dann keine Klärung. Insofern sehe ich da Reformbedarf.

\section{Auch wenn es heute nicht mehr problematisch ist, zu studie- ren und einen Beruf zu ergreifen. Ab einer bestimmten Posi- tion stoßen Frauen an eine Glasdecke. Wie sind Sie damit umgegangen?}

Ich denke, da hat sich sehr viel verändert. Wir haben inzwischen die Gleichberechtigung mit Gesetzen, Gleichstellungs- 
stellen und dergleichen in einer Weise durchsetzen können, dass Diffamierungen, Diskriminierungen und Behinderungen von Frauen, wie sie zu meiner Zeit noch üblich waren, in dem Umfang nicht mehr möglich sind. Ich denke auch, dass sich Frauen das gar nicht mehr gefallen lassen.

In meiner Ausbildungszeit und auch später noch im Beruf musste ich lernen, mich durchzusetzen, wenn ich etwas werden wollte und durfte mich nicht abschieben lassen. Wie man das macht? Man hält einiges aus, möglichst mit Humor und Gelassenheit, reagiert nicht zu schnell empfindlich und beweist sich im Übrigen durch gute Arbeit, Zuverlässigkeit und Kooperationsfähigkeit. Ich hatte auf dem Weg in den Journalismus eine ganze Menge Stolpersteine zu überwinden, weil damals die Haltung vorherrschte, auch bei meinem so genannten Volontärsvater in Hamburg: „Wir wollen hier keine Frauen." Das hat sich dann aber geändert, als ich schon nach anderthalb statt erst nach zwei Jahren in die Redaktion übernommen wurde.

Auf jeden Fall sollte man allen Frauen auf ihrem Weg in den Beruf raten: Wisst, was ihr wollt und bleibt beharrlich. Sagt: Ich will diesen Beruf. Und wenn ihr diese innere Sicherheit habt, dann weicht auch nicht ab von eurem Weg, lasst euch nicht entmutigen!

\section{Wie war die Arbeit als Redakteurin, waren Sie die einzige Frau? Hatten Sie Unterstützung in der Redaktion oder waren Sie auf sich allein gestellt?}

Ich habe 1963 bei der WELT als Volontärin angefangen. Ich war nicht die einzige Frau, es gab einige, die schon über zehn Jahre da waren. Aber die verhielten sich mir gegenüber eher ähnlich wie die Männer, die wollten auch keinen weiblichen Nachwuchs. Also, man kann nicht sagen, dass sich die Frauen mit mir solidarisiert hätten. Insofern war ich schon auf mich allein gestellt.

In der Feuilleton-Redaktion, in der ich dann nach dem Ende des Volontariats gearbeitet habe, bin ich ganz gut klargekommen. Es waren eigentlich nur die Jahre als Volontärin, in denen ich so einiges durchzustehen hatte, wie so eine Art Prüfung, ob ich es auch wirklich durchhalte. Nach der Festanstellung hörten die Schikanen dann auf. Aber ich hatte zum Glück auch einen Fürsprecher, der damals zur Chefredaktion gehörte, Ernst Cramer, dem ich auch zu verdanken hatte, dass ich überhaupt als Volontärin angenommen wurde. Das habe ich ihm nie vergessen. Er gab mir das Gefühl, da ist jemand, den ich mit meinem unbedingten Willen, diesen Beruf ergreifen zu wollen, überzeugen konnte. Er hat mir das zugetraut.

\section{Stellte sich mit der Geburt Ihres Kindes ein Karriereknick ein? Wie sind Sie mit dieser Situation umgegangen? Was würden Sie den Kolleginnen heute empfehlen?}

1968 ist mein Sohn geboren. Da war ich bei der WELT und noch verheiratet. 1971 bin ich geschieden worden. Für mich war klar, dass ich diese Festanstellung nicht mehr weiter halten konnte, weil die Arbeitszeiten überhaupt nicht mit dem Rhythmus eines Kleinkindes übereinstimmten. Ab 1971 arbei- tete ich deshalb freiberuflich. Dieser Übergang hat mich nicht beunruhigt, weil ich mir in den sieben Jahren in der Redaktion ein journalistisches Potential erarbeitet hatte. Außerdem begannen damals in den 70ern die Reformjahre, in denen unentwegt darüber diskutiert wurde, wie man die Gesellschaft und das Recht verändern könnte. Die 68er lagen gerade hinter uns, es war eine sehr aufregende, auch publizistisch interessante Zeit, und ich war sicher, dass ich mich mit meinen juristischen Kenntnissen und meinen Interessen auf dem freien Markt bewähren könnte. Das war dann auch so.

Ich habe zunächst weiter für Zeitungen und Frauenzeitschriften geschrieben, die gesamten Rechtsreformen in Familien- und Fallgeschichten übersetzt und den Leser/innen erzählt, was Neues auf sie zukommt, zum Beispiel beim elterlichen Sorgerecht, beim Eherecht, beim Scheidungsrecht, beim Rentenrecht usw.

Daneben wurde die kindliche Entwicklung als publizistisches Thema zu meinem zweiten Standbein. Ich hatte ja selber ein Kind, habe sehr viel über Pädagogik gelesen und mich auch über Entwicklungspsychologie weiter informiert. Später kamen Bildungsthemen hinzu, darunter auch das damals heiß diskutierte Thema Kinder und Fernsehen. Darüber habe ich dann viele Artikel, Broschüren und auch zwei Bücher geschrieben.

Der Beruf stand für mich - neben meinem Sohn natürlich - immer im Mittelpunkt meines Lebens. Als mein Sohn groß wurde und die Mutter nicht mehr so brauchte, habe ich mich dann noch mehr darauf konzentriert. Ich empfinde das auch als einen großen Reichtum, diese starke Identifizierung mit meinem Beruf. Dass ich ein ganzes Leben lang das machen konnte, was ich wirklich gerne machen wollte und daraus auch meine Kraft und meinen Lebenssinn gezogen habe. Das heißt, ich wollte Menschen und ihre Lebensverhältnisse verstehen lernen und mit meinen Berichten zum Erkennen und Beseitigen von Missständen beitragen.

Ich habe 1989 zum zweiten Mal geheiratet. Mein Mann war Leiter des Landessozialamtes hier in Hamburg und dann Staatssekretär für Soziales im Berliner Senat. Meine Interessen und seine berufliche Arbeit verbanden sich damit auf das Glücklichste.

Was ich den Kolleginnen heute empfehlen würde? Es ist natürlich sehr viel riskanter geworden, auf den freien Markt zu gehen, weil die Konkurrenz rapide zugenommen hat, der Kampf um Aufträge ist härter heute, und die Ausrichtung auf Marktgängigkeit und Quoten bestimmt überwiegend die journalistischen Inhalte.

Eine Journalistin, die sich zutraut, dabei bestehen zu können, ohne zu viele Zugeständnisse machen zu müssen, sollte sich selbständig machen. Denn diese Art der journalistischen Arbeit bietet die meisten Freiheiten. Es ist zwar einerseits schwerer geworden, freiberuflich zu bestehen, andererseits aber auch leichter. Denn es gibt inzwischen sehr viel mehr Gesetze, die die Vereinbarkeit von Familie und Beruf ermöglichen. Diese zahlreichen Hilfen sollten Frauen in jedem Fall in Anspruch nehmen und nutzen - bei gleichzeitiger Bereitschaft, vieles selber zu organisieren. 
Warum haben Sie das Medium gewechselt - von der Zeitung zum Fernsehen? Ging das so einfach? Wie haben Sie Themen gefunden?

Das Medium habe ich ja in dem Sinne gar nicht gewechselt. Ich bin immer schreibende Journalistin geblieben. Es ist nur ständig etwas hinzugekommen. Ich habe zunächst für Zeitungen und Zeitschriften und dann auch Bücher geschrieben, danach ist der Rundfunk dazugekommen. Ich habe die Medien sozusagen durchprobiert. Im Fernsehen habe ich schließlich die Möglichkeit gesehen, nicht nur über das Wort, sondern auch über das Bild etwas auszudrücken.

Mitte der 70er Jahre fing ich dann an, Filme zu machen. Meine ersten Filme hatten noch einen unmittelbaren Bezug zu Familienthemen. „Ich will mein Kind weggeben “ zum Beispiel porträtiert Frauen, die ihr Kind zur Adoption freigegeben

\section{In der DDR waren es vor allem die Lebensverhältnisse und das politische Selbstverständnis, die meine Neugier erregten.}

haben. Es folgten Dokumentationen und Reportagen über junge Partnerschaften, Witwen und Findelkinder. Ende der 70er/Anfang der 80er Jahre interessierten mich dann andere Themenbereiche. Die damalige DDR und Israel zum Beispiel. Das deutsch-jüdische Verhältnis in der Geschichte, das Jahrhundertverbrechen Holocaust mit dem Blick auf die Opfer und die Täter sowie die moderne israelische Gesellschaft und die palästinensischen Verhältnisse haben mich sehr beschäftigt und zu Filmen angeregt.

In der DDR waren es vor allem die Lebensverhältnisse und das politische Selbstverständnis, die meine Neugier erregten.

Nach der Wende wurde mir bei einem Besuch im Bonner Verteidigungsministerium angetragen, „Die Integration der NVA in die Bundeswehr" filmisch zu dokumentieren. Dieses Thema interessierte mich vor allem unter psychologischen Aspekten. Wie kann man Soldaten aus einer bis dahin feindlichen Armee in die eigenen Reihen übernehmen - und was geht in diesen Menschen vor? Müssen sie nicht in Identitätskrisen kommen? Dieser Film wurde nicht nur mit großem Interesse von der Bundeswehr entgegengenommen, sondern daraus entstand dann auch ein Fernsehfilm. Ich habe danach weitere Filme über Bundeswehr-Einsätze auf dem Balkan und in den Ausbildungslagern für diese Einsätze produziert. Dabei haben mich immer in erster Linie die Gedanken und Gefühle der Soldaten bei ihren Er- lebnissen und Erfahrungen interessiert, und genau das habe ich dann den Zuschauern vermittelt.

Jetzt mache ich wieder Filme über Kinder und ihre Entwicklung, denn ich bin seit einigen Jahren Großmutter und zu meinen frühen Interessen zurückgekehrt.

Zur Themenfindung: Es ist eine Frage der Wahrnehmung. Was trifft einen, was nimmt man wahr in verschiedenenen Lebensphasen? Manche Dinge, die andere interessieren, nehme ich gar nicht wahr, weil sie nicht in meinen Lebenskontext passen. Ich finde jedoch immer Themen, die ich sofort aufgreifen und gerne machen würde.

Wann und warum fingen Sie an, sich für Frauenpolitik bzw. feministische Rechtspolitik zu interessieren?

Mit der Reformpolitik der sozial-liberalen Koalition in den 70er Jahren bin ich so richtig politisch aufgewacht. Die Reform des Scheidungsrechts, die Neuerungen beim Unterhalt und der Versorgungsausgleich, das geänderte elterliche Sorgerecht, die Rentendebatte. Damals fingen auch die ersten Diskussionen mit feministischem Hintergrund an, über Abtreibung zum Beispiel. Ich habe damals unter anderem die Dokumentation über den Memminger Abtreibungsarzt gedreht, mit der ich immer noch zitiert werde (Filmtitel: „Mit unerbittlicher Härte“). Es war ein Skandalprozess und der Film zeigte auf, was in unserer Republik passierte. Eine hochspannende Zeit.

Was empfehlen Sie heute für den Beginn einer journalistischen Karriere? Ist ein Jurastudium sinnvoll oder völlig unverzichtbar? Was empfehlen Sie Juristinnen, die sich für eine journalistische Karriere interessieren? Ist ein Volontariat notwendig?

Ich halte im Journalismus ein abgeschlossenes Grundstudium für gut, richtig und wichtig. Damit ist eine solide Fachkompetenz vorhanden. $\mathrm{Ob}$ man nun Jura studiert, Literaturwissenschaften, Psychologie oder Physik ist egal, je nachdem, was man gerne später mal machen möchte. Wissenschaftsjournalismus erfordert natürlich andere Voraussetzungen, als wenn sich jemand für Theaterkritik oder Fernseh-Reportagen interessiert. Und dann sollte eine journalistische Ausbildung folgen, also ein Volontariat. Es gibt heute allerdings viele verschiedene Wege zum Journalismus, so zum Beispiel auch die Journalistenschulen. Manche Kolleginnen kommen als Quereinsteigerinnen dazu, weil sie ihre Berufung überhaupt erst entdecken, wenn sie schon zehn Jahre Rechtsanwältin sind.

Ein gewisses Talent für den Beruf ist natürlich notwendig. Themen so präsentieren zu können, 
dass Leser oder Zuschauer sie mit Interesse und Verständnis aufnehmen. Eine Möglichkeit für Juristinnen ist es auch, über Rechtsreformen Bücher zu schreiben, die Laien verstehen. So hat es meine Kollegin Eva-Marie von Münch mit Erfolg und großer Kompetenz gemacht.

Wann haben Sie den djb kennen gelernt? Wann und warum sind Sie beigetreten? Verbanden Sie mit dem Beitritt bestimmte Erwartungen?

Den Deutschen Juristinnenbund habe ich Anfang der 70er Jahre kennen gelernt. Beigetreten bin ich 1976. Mit meinem Beitritt habe ich auch Erwartungen verbunden, die dahin gingen, in den Kommissionen des Juristinnenbundes zur Verbesserung der Situation der Frauen mitwirken zu können Das habe ich dann auch getan. Die Leiterinnen der Kommissionen wurden in der Politik, in Bundestagsausschüssen, gehört. So hatte ich das Gefühl, ein wenig mit dazu beitragen zu können, dass sich Gesetze zu Gunsten von Frauen, Kindern und Familien entwickeln.

Sie waren von 1987 bis 1989 Vorstandsmitglied. Was waren damals die rechtspolitischen Themen?

Ich war mehrere Jahre Vorstandsmitglied im Bundesvorstand. Die rechtspolitischen Themen waren die Rentenreform, $\mathbb{} 218$, die Gentechnologie, die nichteheliche Lebensgemeinschaft und natürlich auch die Verbesserung des Scheidungsrechtes, die Reform des Unterhaltsrechts, des elterlichen Sorgerechts und anderes. Diese Zeit habe ich als hoch spannend erlebt, weil so viel so Entscheidendes passierte.
Hat Ihnen die Verbandsarbeit weitergeholfen oder aufgrund ihrer Zeitaufwendigkeit eher am Geldverdienen gehindert?

Die Verbandsarbeit hat meinen Horizont erweitert und mir wichtige und gute berufliche und menschliche Kontakte verschafft. Nach dem Zeitaufwand habe ich nicht gefragt. Das war mir nicht wichtig. Die Verbandsarbeit hat mich nicht am Geldverdienen gehindert. Ich habe aufgrund meiner Interessen eine ganze Reihe von Ehrenämtern bekleidet und tue es noch. Das nimmt natürlich Zeit in Anspruch. Aber das habe ich nie bereut, es war und ist alles anregend und sinnvoll. Und wenn es das meiner Meinung nach nicht mehr ist, höre ich damit auf.

Wenn ich auf mein bisheriges Leben als Juristin und Journalistin zurückblicke, muss ich sagen, dass ich ganz viele Chancen hatte und sie genutzt habe. Es ist beruflich und persönlich ein erfülltes Leben. Das ist heute für manche junge Frauen aus meiner Sicht schwieriger zu erreichen, insbesondere die berufliche Erfüllung, weil nicht mehr jede heute das machen kann, was sie wirklich gerne möchte. Und so kann ich mich wirklich glücklich schätzen.

Im Übrigen habe ich aber noch einiges vor. Ich will noch weitere Filme über die kindliche Entwicklung und Bildung machen und wieder mehr schreiben, auch über ganz andere Themen, die eher auf persönlichen Erfahrungen beruhen. Ich glaube, ganz werde ich nie aufhören, in meinem Beruf zu arbeiten, dazu macht er einfach zu viel Spaß - und neugierig bin ich immer noch. Außerdem habe ich einen großen Garten, viele Freunde und zwei wunderbare Enkelkinder, Leben gibt es also genug in meinem Umfeld.

Frau Mundzeck, herzlichen Dank für das Gespräch.

\section{Impressum}

\author{
Schriftleitung: \\ Anke Gimbal, Rechtsassessorin (V.i.S.d.P.) \\ Juliane Lindner
}

Redaktionsanschrift:

Deutscher Juristinnenbund e. V.

Anklamer Str. 38

10115 Berlin

Telefon: 030/ 443270-0

Telefax: 030/ 443270-22

E-Mail: geschaeftsstelle@djb.de

\section{Druck und Verlag:}

Nomos Verlagsgesellschaft mbH \& Co. KG

Waldseestr. 3-5

D-76530 Baden-Baden

Telefon: 07221/ 2104-O

Telefax: 07221/2104-27

\author{
Anzeigenverwaltung und \\ Anzeigenannahme: \\ Sales friendly, Verlagsdienstleistungen \\ Bettina Roos \\ Siegburgerstr. 123 \\ D-53229 Bonn \\ Telefon: 0228/ 97898-0 \\ Telefax: 0228/ 97898-20 \\ E-Mail: roos@sales-friendly.de
}

Die Zeitschrift sowie alle in ihr enthaltenen einzelnen Beiträge und Abbildungen sind urheberrechtlich geschützt. Jede Verwertung, die nicht ausdrücklich vom Urheberrechtsgesetz zugelassen ist, bedarf der vorherigen Zustimmung des Verlages.

Namentlich gekennzeichnete Artikel müssen nicht die Meinung des Herausgebers oder der Schriftleitung wiedergeben. Unverlangt eingesendete Manuskripte - für die keine Haftung übernommen wird - gelten als Veröffentli- chungsvorschlag zu den Bedingungen des Verlags. Es werden nur unveröffentlichte Originalarbeiten angenommen. Die Verfasser erklären sich mit einer nicht sinnentstellenden redaktionellen Bearbeitung einverstanden.

\author{
Erscheinungsweise: \\ vierteljährlich \\ Bezugspreis 2008: \\ jährlich 48,-€, Einzelheft 13,-€
}

Alle Preise zzgl. Vertriebs-Direktbeordnungsgebühren inkl. MWSt.;

Bestellungen nehmen entgegen:

Der Buchhandel und der Verlag; Kündigung jeweils drei Monate zum Kalenderjahresende. Zahlungen jeweils im Voraus an: Nomos Verlagsgesellschaft, Postbank Karlsruhe, Konto 7363651 (BLZ 66010075) oder Stadtsparkasse Baden-Baden, Konto 5002266 (BLZ 66250030). 